\section{Non-enhancing gliomas: does intraoperative ultrasonography improve resections?}

\author{
Aliasgar V. Moiyadi, Prakash Shetty, Robin John \\ Division of Neurosurgery, Department of Surgical Oncology, Tata Memorial Centre, \\ Homi Bhabha National Institute, Mumbai, India
}

Purpose: Non-enhancing diffuse gliomas are a challenging surgical proposition. Delineation of tumor extent on preoperative imaging and intraoperative visualization are often difficult.

Methods: We retrospectively analyzed all cases of non-enhancing gliomas that were operated on using navigated 3-dimensional ultrasonography (US). Tumor delineation (good, moderate, or poor) on preoperative magnetic resonance imaging (MRI) and intraoperative US was compared. Post-resection US findings with respect to residual tumor status were compared to the postoperative imaging findings. The extent of resection was calculated and recorded.

Results: There were 55 gliomas (43 high-grade, 12 low-grade). Forty were close to eloquent areas. The pre-resection concordance of MRI with US was $56 \%$, with US defining more tumors as well-delineated $(n=26)$ than MRI $(n=13)$. US was used for resection control in 50 cases. Gross tumor resection was achieved in 24 cases (51\%). US correctly predicted the residual tumor status in $78 \%$ of cases. The use of US led to radical resections even in some tumors preoperatively deemed to be unresectable. However, eloquent location was the only independent predictor of the extent of resection.

Conclusion: Intraoperative US is a useful tool for guiding resection of non-enhancing gliomas. It may be better than MRI for delineating these tumors, and may thereby facilitate improved resection of these otherwise poorly delineated tumors. However, functional boundaries remain the main limiting factor for achieving complete resection of non-enhancing gliomas.

Keywords: Intraoperative ultrasound; Navigated ultrasound; Non-enhancing gliomas;

Predictive value

\section{Introduction}

Non-enhancing gliomas comprise predominantly low- to intermediate-grade diffuse gliomas (World Health Organization grades 2 and 3). Although their histology is varied, the spectrum of diffuse fibrillary astrocytomas (grade 2) and anaplastic astrocytomas (grade 3) predominates in this group. Regardless of the histology, at presentation most patients undergo surgical resection. The goal of surgery is to maximize the resection, which in turn provides both symptomatic and oncological benefits. Therefore, optimization of the resection remains a priority for neurosurgeons involved in the

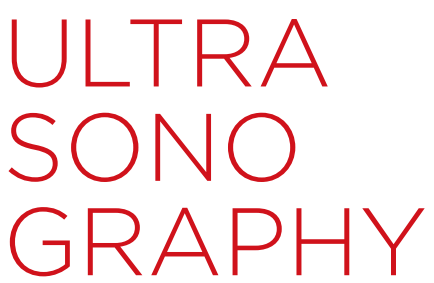

ORIGINAL ARTICLE

https://doi.org/10.14366/usg. 18032 pISSN: 2288-5919 • elSSN: 2288-5943

Ultrasonography 2019;38:156-165

Received: May 31, 2018

Revised: July 28, 2018

Accepted: July 29, 2018

Correspondence to:

Aliasgar V. Moiyadi, MCh, Division of Neurosurgery, Department of Surgical Oncology, Tata Memorial Centre, Homi Bhabha National Institute, Mumbai 400012, India

Tel. +91-22-24177279

Fax. +91-22-27405061

E-mail: aliasgar.moiyadi@gmail.com

This is an Open Access article distributed under the terms of the Creative Commons Attribution NonCommercial License (http://creativecommons.org/ licenses/by-nc/4.0/) which permits unrestricted noncommercial use, distribution, and reproduction in any medium, provided the original work is properly cited.

Copyright (C) 2019 Korean Society of Ultrasound in Medicine (KSUM)

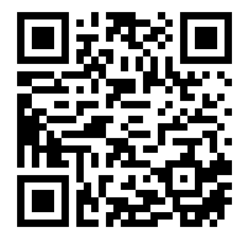

How to cite this article:

Moiyadi AV, Shetty P, John R. Non-enhancing gliomas: does intraoperative ultrasonography improve resections? Ultrasonography. 2019 Apr;38(2):156-165. 
management of these lesions. The surgical strategy is typically twopronged. Adequate visualization of the tumor intraoperatively and reliable control of the resection is essential to maximize the resection extent. Simultaneously, functional mapping and monitoring is crucial for ensuring neurological integrity. Despite the use of an operating microscope, visualizing the tumor and delineating its boundaries can be very challenging. Resection rates are variable and often less than anticipated [1], even in glioblastomas (which are often better circumscribed on imaging than non-enhancing gliomas), with the surgeon incorrectly predicting gross total excision (overestimating) in over two-thirds of cases. Often this is because of "missed" remnants due to inadequate discrimination from normal tissue. For malignant gliomas that are predominantly contrast-enhancing, it is possible to use intraoperative fluorescence to improve tumor visualization $[2,3]$. However for non-enhancing gliomas, this is not currently an option. To maximize and guide resections, therefore, other forms of intraoperative imaging are necessary. Although intraoperative magnetic resonance imaging (MRI) is the gold standard, logistics (cost and infrastructure) make intraoperative MRI difficult at most centres. Intraoperative ultrasonography (US) is emerging as a useful alternative to intraoperative MRI and may be very well suited for non-enhancing gliomas. Our strategy has been to use navigated 3-dimensional US (n3DUS). We have earlier reported our experience with this technique in malignant gliomas [4]. This present report describes our experience with this technique in non-enhancing gliomas, focusing on its utility and role for these tumors.

\section{Materials and Methods}

This was a retrospective analysis of prospectively collected data that received approval from the institutional review board with a waiver of consent as per institutional policies. All non-enhancing gliomas (based on MRI) that were operated on using intraoperative n3DUS between June 2011 and May 2016 were included. The inclusion criteria for the present analysis were nonenhancing supratentorial intra-axial lobar tumors, for which intraoperative US was used, and for which all archival US images were available for review. We used the SonoWand intraoperative US system (SonoWand AS, Trondheim, Norway). The system allows the use of navigated US in conjunction with preoperative MRI findings. Multiple probes were used as required (8FPA probe [3-8 $\mathrm{MHz}$ phased array, $25 \times 17 \mathrm{~mm}$ footprint], 10FPA-MC [5-10 MHz phased array, $15 \times 13$ $\mathrm{mm}$ footprint], and 12FLA [6-12 MHz linear array, $32 \times 11 \mathrm{~mm}$ footprint]). Routine microsurgical techniques were used for tumor resection. Intraoperative functional mapping was conducted when tumors were close to eloquent areas. Surgical decisions were based on a combination of US findings with functional information and the surgeon's clinical judgement. The hospital electronic medical records and picture archiving and communications system were reviewed to extract clinicoradiological data. Resectability was routinely recorded prospectively in the preoperative notes of all cases operated on using US, and tumors were classified as either resectable or unresectable. Resectability is usually very difficult to define objectively, but as used in our practice and in this study, resectable tumors were those where a potential complete resection was anticipated and planned preoperatively. Preoperative MRI (done in all cases) was used to classify tumors as well, moderately, or poorly delineated based on the ability to resolve the interface between the tumor and the normal brain, as well as the ability to delineate the margin all around the tumor, as described elsewhere (Table 1, Fig. 1) [5]. Eloquence was defined based on earlier published criteria (Sawaya grade I, non-eloquent; grade II, close to eloquent; and grade III, involving eloquent areas) [6]. Intraoperative US findings from a prospectively maintained operative database were also reviewed. These findings included details about various technical parameters, as well as the US characteristics of the tumors. The US findings specifically included the delineation of the tumor (categorized as good, moderate and poor, similar to the MRI classification) (Table 1), and the utility of the intraoperative US for dictating the course of the resection (number of scans per patient, decision to resect further, and the status of the final residue). Postoperative MRI scans were also reviewed to document the extent of resection (EOR). Residual tumor extent was assessed by comparing T2 and fluid-attenuated inversion recovery (FLAIR) signals on preoperative and postoperative scans. EOR was classified semi-quantitatively as gross total resection (GTR) (complete resection of all T2 and FLAIR changes), near total resection (NTR) (90\% - 99\% resection), subtotal resection (STR) (50\%-90\% resection), and partial resection (PR) ( $<50 \%$ resection). The concordance between post-resection (intraoperative) US and postoperative MRI findings was assessed, and the accuracy and

Table 1. Classification of tumors based on magnetic resonance imaging and ultrasound characteristics

\begin{tabular}{lll}
\hline & \multicolumn{1}{c}{ Margin } & \multicolumn{1}{c}{ Demarcation from surrounding parenchyma } \\
\hline Well delineated & Well defined all around & Easily demarcated \\
Moderately delineated & Well defined focally but unclear in most places & Easily demarcated \\
Poorly delineated & Unclear & Difficult to distinguish the interface of the tumor with the normal brain \\
\hline
\end{tabular}


predictive value of US were calculated.

\section{Statistical Analysis}

All categorical data were expressed as frequencies and percentages. Resectability and the EOR may be influenced by various factors.
These factors (MRI delineation, US delineation, location relative to eloquent areas, prior treatment, and tumor histology) were evaluated by the chi-square test and subsequently in a multivariate model. For the purpose of our analysis, the factors were dichotomized, with moderate and poor delineation (both for MRI and US) grouped

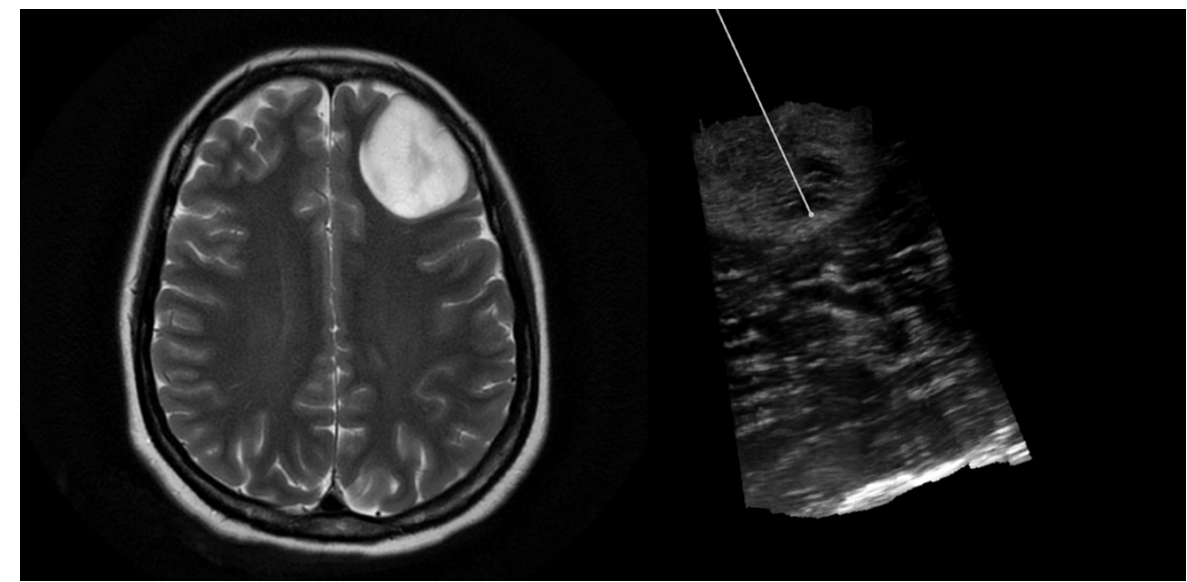

A

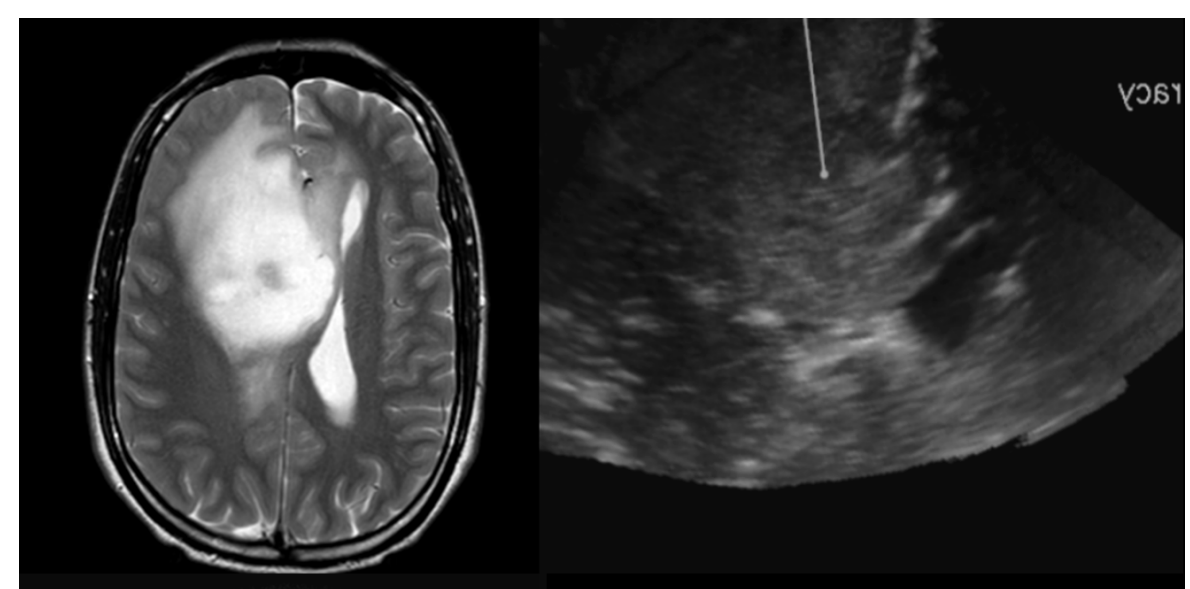

B

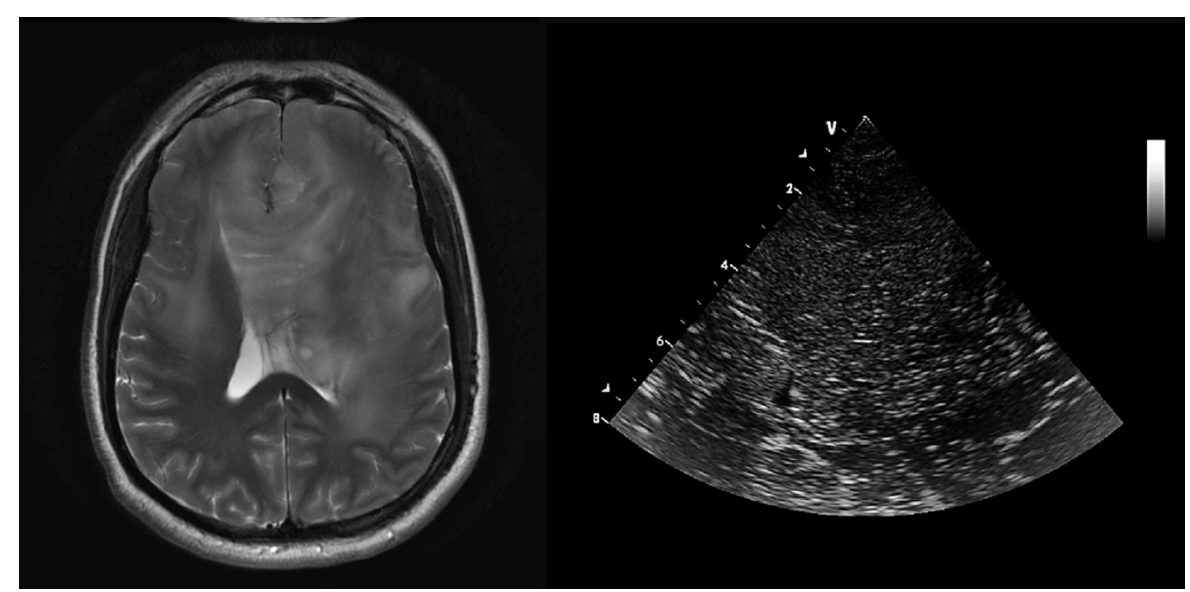

C
Fig. 1. Representative images showing the various types of tumor delineation on magnetic resonance imaging (MRI) and ultrasonography (US).

The left column shows T2-weighted MRI, and the corresponding intraoperative image is shown on the right panel. A. Both MRI and US in a 30-year-old woman with a left frontal astrocytoma show a welldelineated tumor with distinct margins. B. Images of a 30-year-old man with a right frontal anaplastic astrocytoma with moderate delineation and blurring of tumor margins on both MRI and US are shown. C. Images of a 42-year-old man with a diffuse frontotemporal glioma are shown. Note the poor demarcation of the tumor-normal brain interface on both MRI and US. Note the poor demarcation of the tumor-normal brain interface on both MRI and US. 
together, and eloquent regions II and III grouped together as eloquent. EOR was dichotomized as GTR versus non-GTR. A P-value of less than 0.05 was considered to indicate statistical significance. A penalized maximum likelihood estimator for the logistic regression model using the Firth correction was used due to problems of separation and multicollinearity for the selection of variables for the multivariate analysis. All statistical analyses were carried out using STATA ver. 14.0 (StataCorp, College Station, TX, USA).

\section{Results}

During the study period, n3DUS was used in 66 nonenhancing gliomas. Eleven cases were excluded from the present analysis for various reasons (4 were non-lobar tumors, 4 had no archival US images, and in 3 cases US could not be used due to technical reasons). Thus, 55 cases of non-enhancing gliomas were included in the present analysis. The demographic and clinical profile of the patient cohort is shown in Table 2. More than half of the tumors were deemed resectable (56\%). Navigated US was used along with preoperative navigated MRI in 12 cases and as a stand-alone independent modality in the remaining 43 cases. Some of these cases were part of an earlier study describing the technique of "direct" navigated US [7]. A linear probe (FLA) was used in 15 cases and a phased array probe (8-FPA) in 40 cases. Histologically, the majority of tumors ( $n=43,77 \% ; 42$ anaplastic astrocytomas and 1 glioblastoma) turned out to be high-grade gliomas, while only 12 $(22 \%)$ were diffuse astrocytomas (grade 2 ).

Table 2. Demographic and radiological features of the patient cohort

\begin{tabular}{lc}
\hline & No. $(\%)(\mathrm{n}=55)$ \\
\hline Age, mean (range, yr) & $36.5(8-64)$ \\
Sex (male/female) & $35 / 20(64: 36)$ \\
Prior treatment & $10(18)$ \\
Yes & $45(82)$ \\
No & \\
Intent of surgery & $53(96)$ \\
Radical & $2(4)$ \\
Biopsy & \\
Eloquent location & $15(27)$ \\
I (non-eloquent) & $23(42)$ \\
II (close) & $17(31)$ \\
III (eloquent) & \\
Resectability & $31(56)$ \\
Resectable & $24(44)$ \\
Unresectable &
\end{tabular}

In five cases, US was used only for localization of the lesion (essentially for obtaining a frameless biopsy in two cases and for limited debulking in 3). In the remaining 50 cases, it was used for resection control. Repetitive US scans were performed 1-4 times in each of these cases (mean, 2 scans per case). There was no relationship between the number of scans obtained and the delineation of the tumors on US. The intermediate scans prompted further resection in 42 of the cases (84\%) where it was used for resection control. Postoperative neurological worsening was seen in nine cases $(16 \%)$. In two of these patients, this was transient, whereas the remaining seven had prolonged neurological deficits (12\%).

Pre-resection MRI and US tumor characteristics were assessed in all 55 cases (Table 3). Based on MRI morphology, most of the tumors were moderately delineated (32 of 55, 58\%). On US, the tumors were typically hyperechoic with variable internal echogenic signals. Individual tumor morphology was not analyzed in this report. US defined more tumors as well-delineated (26 of 55 ) than did MRI (13 of 55). Almost half (15 of 32) the tumors defined as moderately-delineated on MRI were actually well-delineated on US (Table 3). The proportion of well-delineated tumors in eloquent and non-eloquent areas was the same for US and MRI, though the absolute number of eloquently located tumors in the US-defined group was higher (16 eloquent, 10 non-eloquent) than in the MRIdefined group (8 eloquent, 5 non-eloquent). Fig. 2 shows typical images of a glioma that appeared moderately delineated on MRI but had very clear margins on US, and Fig. 1A shows a glioma that was well-delineated on both US and MRI. The concordance between the US and MRI findings with respect to initial (pre-resection) tumor delineation was $56 \%$ (31 of 55). In addition to tumor morphology, US was very useful for studying adjacent anatomical structures and provided vascular landmarks via color Doppler US (Fig. 3).

We evaluated the accuracy of US for predicting the residual tumor and resection control $(n=47)$. Postoperative MRI was obtained in 47 of the 50 cases where US was used for resection control. The EOR was calculated and analyzed in these 47 cases. After resection, US showed no residue in 19 cases, whereas resection was stopped in the other 28 cases despite residues shown on US because of

Table 3. Comparison of delineation on MRI versus US $(n=55)$

\begin{tabular}{lccc}
\hline \multirow{2}{*}{ MRI delineation } & \multicolumn{3}{c}{ US delineation } \\
\cline { 2 - 4 } & Good & Moderate & Poor \\
\hline Good & $11^{\text {a) }}$ & 2 & 0 \\
Moderate & 15 & $17^{\text {a) }}$ & 0 \\
Poor & 0 & 7 & $3^{\text {a) }}$ \\
\hline
\end{tabular}

MRI, magnetic resonance imaging; US, ultrasonography.

${ }^{a)}$ Concordant cases. 
involvement of eloquent areas or extremely diffuse tumor extent. Based on postoperative MRI, GTR was achieved in 24 cases (51\%), NTR in $10(22 \%)$, STR in $12(25 \%)$, and PR in one (2\%) (Table 4). In the resectable tumors $(n=30)$, the GTR and NTR rates were $70 \%$ and $17 \%$, respectively. In the unresectable tumors ( $n=17), 53 \%$ had
STR. Interestingly, there were still three cases of GTR (18\%) and five cases of NTR $(29 \%)$ in this group.

We specifically compared the presence of a residual tumor on post-resection US with the postoperative MRI-detected residue. The sensitivity, specificity, positive predictive value, and negative

Table 4. Extent of resection and the resectability of the tumors $(n=47)$

\begin{tabular}{lcccc}
\hline & Gross total resection & Near total resection & Subtotal resection & Partial resection \\
\hline Resectable & 21 & 5 & 3 & 1 \\
Unresectable & 3 & 5 & 9 & 0 \\
Total & 24 & 10 & 12 & 1 \\
\hline
\end{tabular}

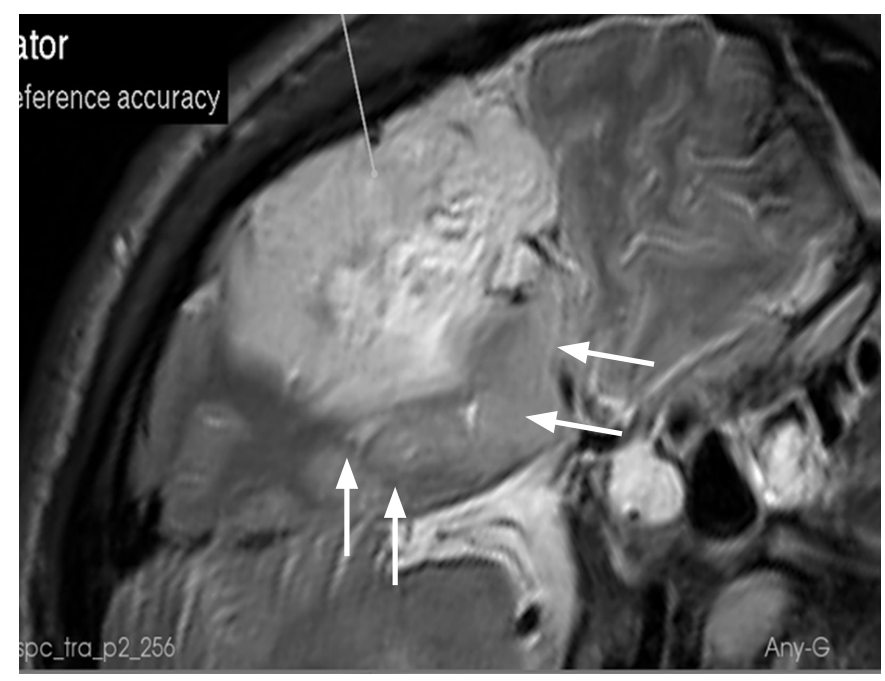

A

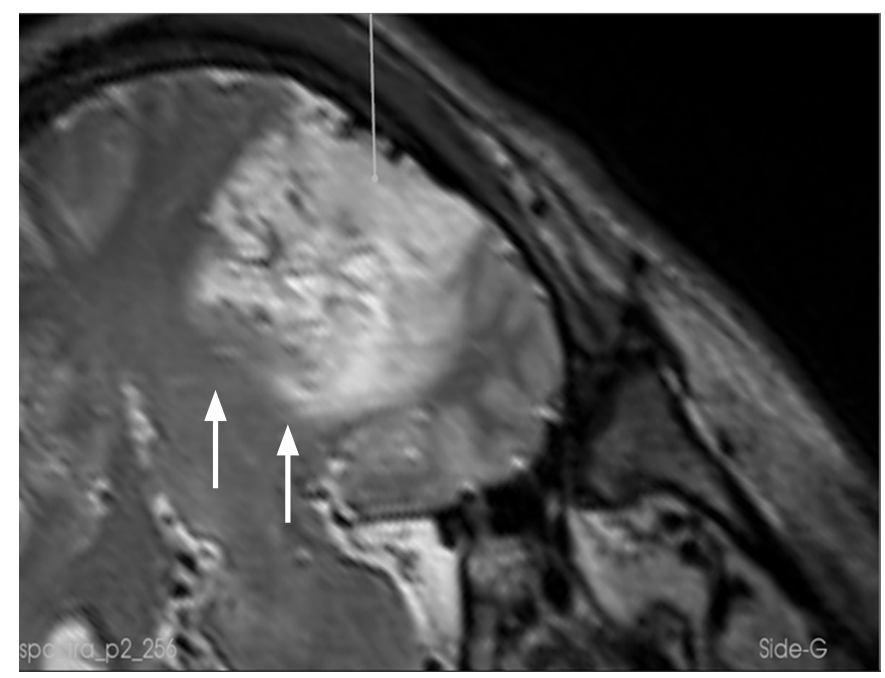

C

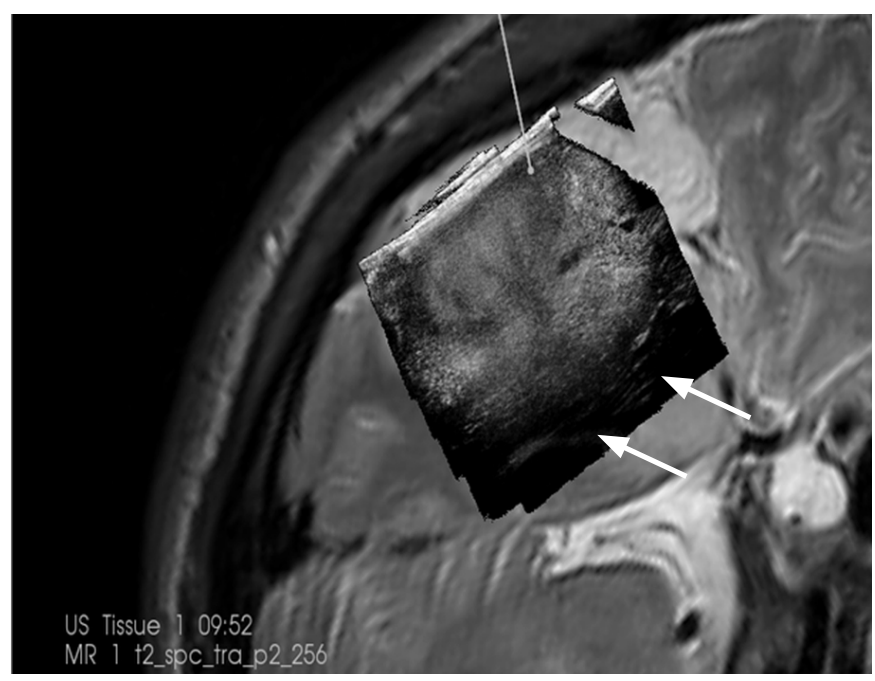

B

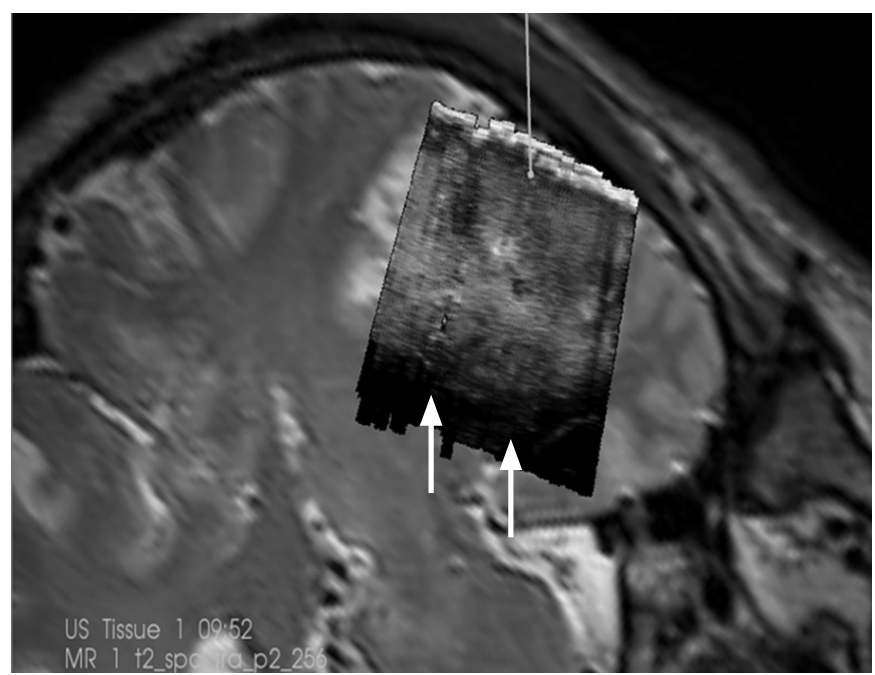

D

Fig. 2. A 34-year-old man with a left temporal anaplastic oligodendroglioma.

A, C. Intraoperative navigated T2-weighted magnetic resonance imaging (MRI) findings in two planes are shown. B, D. An overlay of intraoperative ultrasonography (US) in the same planes as the MRI is shown. Note that the deeper margins of the tumor on MRI (arrows) appear hazy, whereas on US they appear sharper. 


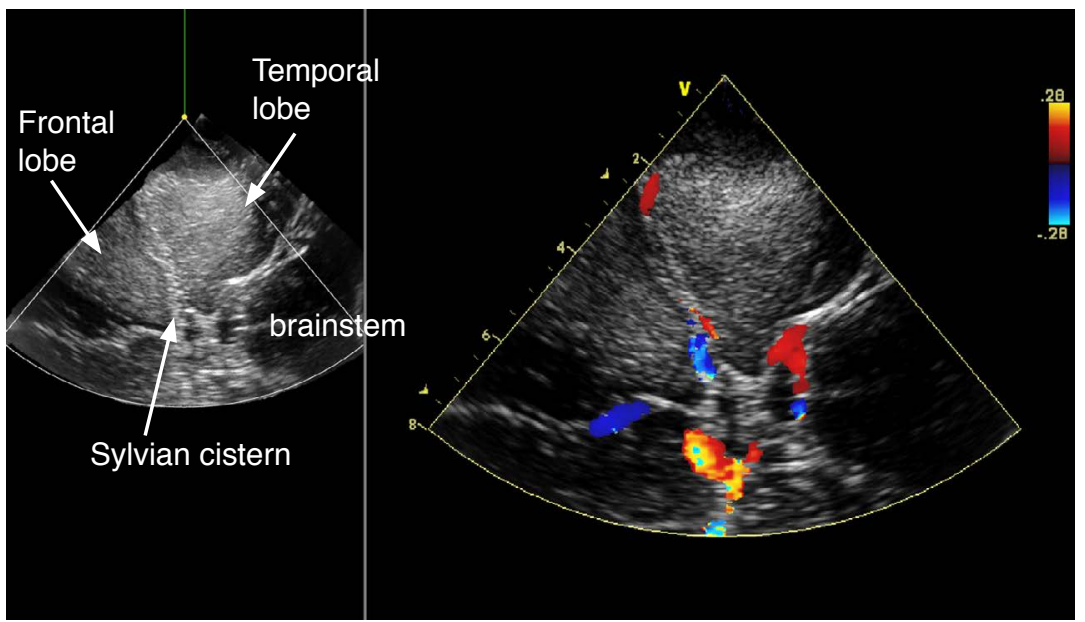

A

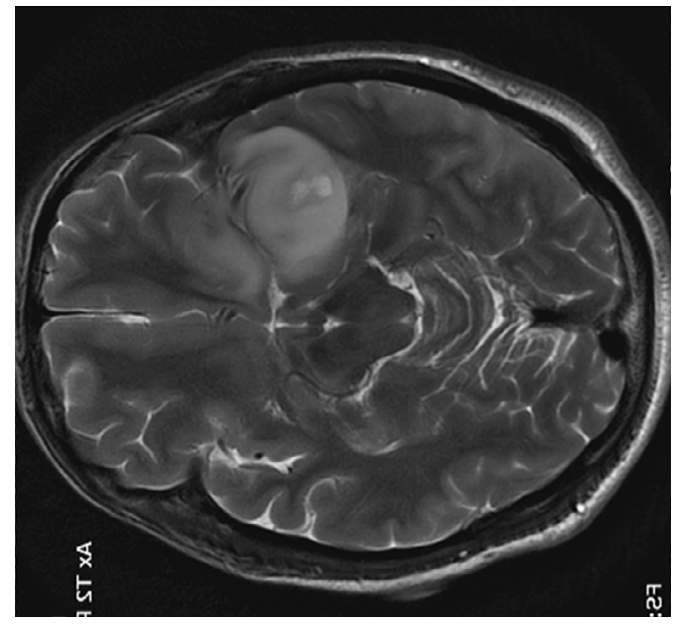

B

Fig. 3. Intraoperative ultrasonography (US) of a 42-year-old woman with a frontotemporal glioma.

A. The native US is seen on the left, and the overlaid color Doppler US on the right. The US also shows sharp, well-delineated margins. Additional real-time vascular information was provided by the intraoperative color Doppler US. B. The corresponding axial T2-weighted magnetic resonance image rotated to be aligned with the US plane is shown.

Table 5. Significance of factors influencing the extent of resection and resectability in the univariate analysis

\begin{tabular}{lcc}
\hline & \multicolumn{2}{c}{ P-value } \\
\cline { 2 - 3 } & Resectability & Extent of resection \\
\hline Eloquence (non-eloquent) & 0.142 & 0.013 \\
MRI delineation (good) & $<0.001$ & 0.050 \\
US delineation (good) & 0.023 & 0.187 \\
Histology (LGG) & 0.878 & 0.669 \\
Prior treatment (yes) & 0.059 & 0.012 \\
\hline
\end{tabular}

MRI, magnetic resonance imaging; US, ultrasonography; LGG, low-grade glioma.

predictive value of US for detecting the residue were $88 \%, 70 \%$, $75 \%$, and $84 \%$ respectively. Overall, the concordance between US and postoperative MRI in predicting the presence of a residual tumor was 78\% (37 of 47; positive concordance in 21 cases and negative concordance in 16 cases). There were 7 US false-positives and 3 US false-negatives.

We analyzed resectability, the EOR, and the factors influencing them. On univariate analysis, tumor delineation on MRI and US, as well as prior treatment, significantly influenced resectability (Table 5). However, EOR was significantly influenced by delineation on MRI, a non-eloquent location, and prior treatment, but not by the US delineation of the tumor. On multivariate analysis, only good MRI delineation significantly influenced resectability (Table 6). GTR was significantly more common in well-delineated tumors on MRI, in those located in non-eloquent areas, and in those with prior treatment. On multivariate analysis, only location in a non-eloquent
Table 6. Multivariate analysis of factors influencing resectability and extent of resection

\begin{tabular}{llll}
\hline & OR & 95\% Cl & P-value \\
\hline Resectability & & & \\
MRI delineation (good) & 0.06 & $0.01-1.03$ & 0.052 \\
US delineation (good) & 0.43 & $0.12-1.58$ & 0.211 \\
Prior treatment received & 2.82 & $0.45-17.72$ & 0.273 \\
Extent of resection (GTR) & & & \\
MRI delineation (good) & 0.32 & $0.06-1.61$ & 0.167 \\
Non-eloquent Location & 0.14 & $0.03-0.66$ & 0.011 \\
Prior treatment received & 7.90 & $1.09-57.39$ & 0.058 \\
\hline
\end{tabular}

$\mathrm{OR}$, odds ratio; $\mathrm{Cl}$, confidence interval; $\mathrm{MRI}$, magnetic resonance imaging; $\mathrm{US}$, ultrasonography; GTR, gross total resection.

area and prior treatment were significantly associated with GTR (Table 6).

\section{Discussion}

Our results show that non-enhancing gliomas remain a challenge in terms of achieving GTR. A significant proportion of such tumors may involve eloquent areas (more than $70 \%$ in our series), precluding a radical resection. Another important reason is the diffuse nature of these tumors, with a large percentage of them (78\%) not being well-delineated on MRI. Using intraoperative n3DUS, we were better able to delineate many of these tumors (almost 3 times the number that could be well-defined on MRI), enabling GTR in $51 \%$ of all such 
non-enhancing tumors. As expected, the GTR rate was much higher in tumors in non-eloquent regions (73\%) than in those that were close to or involved eloquent regions (20\%). The accuracy of US in correctly predicting the resection status was $78 \%$ when compared to the control MRI done postoperatively.

Objective assessment of the extent of non-enhancing gliomas is difficult because of the diffuse nature of such tumors and the difficulty in accurately delineating true tumor extent on MRI. Whereas FLAIR and T2 signal intensities, if accurately quantified, can reliably predict tumor boundaries in low-grade gliomas, the same cannot be said about higher-grade non-enhancing gliomas where perilesional oedema is extremely difficult to distinguish from the infiltrating tumor front. As in our study, non-enhancing gliomas can include a significant proportion of high-grade lesions (70\%), although other recent studies evaluating the resection of nonenhancing gliomas have reported lower proportions (20\%-40\%) $[8,9]$. Regardless of the eventual histological grade, intraoperatively the neurosurgeon has to rely on visual and tactile cues, as well as imaging adjuncts, to define the tumor boundaries and to optimize the surgical resection. Of note, contrary to some previously published reports [5], in our study US defined more tumors as being welldelineated (twice as many) than MRI. One of the possible reasons is that US and MRI use different physical properties of the tissue being imaged, which could lead to differences in their delineation (MRI uses magnetization of particles, whereas US uses conduction of sound waves in tissues). Other studies, using meticulous pathological correlation of border-zone biopsies, have shown that US is as good, if not better than, MRI in delineating low-grade (nonenhancing) tumors [10]. In addition to better delineation of lowgrade tumors, US is better able to differentiate peritumoral oedema, thereby rendering the true tumor boundaries more clearly (Fig. 1). In the present study, the concordance between MRI and US was only $54 \%$, implying that almost half the time, the US and MRI definitions of tumor boundaries differed. Though our study did not co-register MRI with US in all cases, nor did we perform histological sampling to confirm tumor extent, extrapolating from the previous study by Unsgaard et al. [10], which used the same technology of n3DUS, we can assume that US better delineated the non-enhancing tumor extent. A possible limitation in our study is that the definition of the tumor boundaries was subjective, which could introduce bias into the results. Nonetheless, we used the same criteria for defining tumor extent on MRI and on US, which would minimize such bias.

EOR has been shown to correlate with the prognosis of both high-grade and low-grade gliomas [11,12]. Recently introduced intraoperative fluorescence visualization techniques have significantly improved the resection rate in enhancing malignant gliomas $[2,3]$. For non-enhancing tumors, however, these techniques are not as useful. Various multimodal strategies have been proposed for resecting non-enhancing gliomas. Most of them utilize a combination of functional navigation (using MRI-based functional information) and intraoperative electrophysiological mapping $[13,14]$. Given that a large proportion of such tumors are close to eloquent areas of the brain, the ability of mapping through intraoperative electrophysiological stimulation to improve resection is beyond doubt [15]. Though reliance on only functional mapping has been propounded in the technique of supra-maximal resection by some authors [16], a dual-pronged approach using a combination of intraoperative imaging and functional mapping is the most widely accepted strategy. Intraoperative MRI is generally considered the gold standard for intraoperative imaging tools. However, intraoperative MRI is not very widely available. US, in contrast, is almost ubiquitous in most neurosurgical departments. The role of intraoperative US as a tool for resection control has been extensively studied in gliomas [4,17-22]. Most reports have included either high-grade or low-grade gliomas (or a combination of the two). No previous report has focused exclusively on nonenhancing gliomas as a radiologically distinct group. The challenge with non-enhancing gliomas (irrespective of whether they ultimately turn out to be low- or high-grade), is the difficulty in delineating tumor extent, both preoperatively and intraoperatively. Using navigated US, we were able to achieve an overall GTR rate of $51 \%$ in this group of non-enhancing gliomas. These figures are similar to our earlier experience in malignant gliomas as a histological group [4]. Moreover, the morbidity with this strategy was comparable to that reported in a large series of gliomas [23].

The predictive value of intraoperative US is crucial in supporting its role as a tool for resection control. With appropriate technique and care, artefacts can be minimized and optimal image resolution can be achieved for the purpose of resection control. Post-resection US and postoperative MRI were concordant in $78 \%$ of cases. This was higher than the preoperative concordance. Most of the discordance (8 of the 10 discordant cases) occurred in moderatelydelineated tumors as defined by MRI features. This most likely reflects the difficulty in defining the boundaries of such tumors. In our experience, US better delineated such tumors. Both falsepositives and false-negatives were seen with US. This clearly means that the accuracy of US in predicting tumor residue needs to be improved. However, it could also be argued that MRI may not be the ideal 'gold standard' with which to compare US. It is widely acknowledged that postoperative MRI may not be able to accurately delineate non-enhancing tumor residue. The presence of oedema, ischaemia, and postoperative artefacts in the pericavity area may be confounding factors leading to false positives on MRI itself [24]. In fact, early postoperative MRI may overestimate residual 
tumor extent, which could account for the false negatives seen in the control US [8]. Meticulous interpretation of postoperative MRI with multiple sequences (preferably co-registered preoperative and postoperative MRI) is the ideal way to minimize this error [9]. Nonetheless, this may be difficult in routine practice. Delayed MRI has been suggested to be more reliable for assessing the true residual tumor extent [8]. However, this is not useful for resection control when reliable imaging is required intraoperatively. In such a situation, it is very likely that US can better delineate tumor extent and may be more reliable. US itself has its limitations with artefacts that can compromise its validity, especially during the course of the resection. We did not directly evaluate the quality of US during resection procedures. Various strategies to minimize artefacts can improve the predictive efficacy of US [25]. Practically, we aim to eliminate all blood and debris within the cavity and to ensure optimal acoustic coupling by adjusting the position of the cavity (and the patient) during each procedure. Further refinements in scanning technology and techniques are likely to address this issue in the near future.

Generally, resectability is very difficult to define objectively [1]. Orringer et al. [1] showed that in patients deemed to have resectable tumors, GTR was actually achieved in only $23.5 \%$. Further, the intraoperative impression of GTR was correct in only $30 \%$ of cases. This was a particular problem when the EOR rates exceeded $90 \%$ (i.e., the surgeon's intraoperative estimate may be especially flawed when the EOR approaches $90 \%$ ). Therefore, for realizing the goal of true GTR, some form of intraoperative guidance is needed, especially in the "final stretch." Generally, the preoperative imaging characteristics and proximity to eloquent structures dictates whether a glioma can be radically resected. However, many other subjective factors may influence this assessment. One such variable is the surgeon's intent and mindset. For example, a surgeon with a pre-decided notion that only debulking is adequate may never achieve a radical resection, even in a potentially resectable tumor. For such a surgeon, the tumor would always remain unresectable. It has been shown that when the intent is radical, the overall radicality of the eventual operation is also better [17]. This, coupled with the knowledge that increasing the EOR (even if it falls short of GTR) confers an incremental prognostic benefit [26], makes it imperative to have a radical (albeit realistic and cautious) mindset when dealing with all such potentially resectable gliomas. In such a situation, the preoperative imaging features remain the most important determinants of resectability. Expectedly, in our analysis, resectability was closely correlated with the MRI characteristics of the tumor. It was not correlated with the US delineation, although a significant relationship was found in the univariate analysis. This may be because in routine practice, resectability is judged based on available preoperative imaging, which is almost always MRI, and US is only available later as an intraoperative tool. In fact, our findings suggest that if US is used to decide resectability, more tumors could be deemed resectable. More surprisingly, resectability was not correlated with eloquent location. We did not include functional MRI information in this analysis, which could partly explain the lack of an association. Further, in our practice, we believe that even for eloquent-region tumors, resectability is not precluded based only on preoperative imaging, as intraoperative monitoring/mapping conclusively dictates the resection. Therefore, the "potentially resectable" group, as defined by US characteristics, included many more eloquently-located gliomas than the MRI-defined well-delineated group. Not surprisingly, the eventual EOR was significantly correlated with eloquent location in the multivariate analysis. Neither the MRI nor the US features of the tumor were associated with the EOR. US classified many more tumors as welldelineated than did MRI. This larger group of well-delineated tumors may have included many more tumors in an eloquent area, reducing the GTR rate in this subgroup and therefore making the association of GTR with US delineation weaker than the association of GTR with MRI delineation. Further, as discussed earlier, postoperative MRI itself has limitations in defining the true residue, and using MRI as the gold standard for evaluating the accuracy of US may not have been perfectly accurate. Patients undergoing a reoperation were more likely to have GTR. This reflects selection bias because patients are chosen very carefully when it comes to recurrent tumors, but not necessarily for primary tumors, for which surgery is generally offered to all patients.

In our series, GTR was achieved in $70 \%$ of the tumors deemed resectable. However, in the unresectable group, GTR/NTR was still possible in $47 \%$ of cases. This could be attributed to the use of intraoperative US, along with a combination of functional mapping techniques. A recent study reported that in tumors deemed unresectable, radical ( $>95 \%$ ) resection could still be achieved in up to $42 \%$ of cases [27]. This study focused on the accurate intraoperative verification of eloquent sites, which could lead to more extensive resection. Our study focussed on accurate intraoperative delineation, although we also used mapping when tumors were close to eloquent areas. Jointly, these considerations underscore that we cannot presume that a tumor is unresectable based purely on preoperative information. It substantiates our findings that tumor delineation was better with US, and therefore justifies the conclusion that the use of intraoperative adjuncts may influence the eventual EOR, regardless of the determined resectability.

This analysis has certain limitations. As we have mentioned, the classification of tumor delineation was subjective, with possible 
inter-observer variability, especially in the moderately delineated group. However, applying the same criteria in each case for both MRI-based and US-based delineation accounted for a major source of bias. Another limitation was that post-resection US and MRI were not co-registered. Therefore, an accurate spatial correlation of USbased and MRI-based residue was not possible. Furthermore, the EOR was not determined by volumetric methods, and hence may not have been completely accurate. Moreover, as the location of eloquent areas is the main limiting factor for radical resections, separating the effect of eloquent location from the actual effect of using US is extremely difficult, even with multivariate analysis. Finally, there was no control group (similar tumors resected without the use of US), and hence these results need to be validated in further studies. Nonetheless, the fairly large number of cases in this series (for a cohort of non-enhancing gliomas) and the use of a high-end navigated US system with meticulous documentation and correlation with postoperative imaging provide a reliable assessment of the role of US in the resection of non-enhancing gliomas.

In conclusion, non-enhancing gliomas remain a difficult group of tumors to address surgically, primarily because of the difficulty in delineating these tumors adequately. The preoperative MRI appearance of the tumor reliably predicts their resectability. US improves the delineation of the tumor, and also provides updated information during the course of the resection, and therefore probably facilitates more radical resection, even in so-called unresectable tumors. Nonetheless, certain tumors, even if betterdelineated on US, may not be amenable to GTR due to eloquent location.

ORCID: Aliasgar V. Moiyadi: https://orcid.org/0000-0002-4082-2004

\section{Conflict of Interest}

No potential conflict of interest relevant to this article was reported.

\section{Acknowledgments}

The authors would like to thank Mrs Sadhana Kannan, from the Department of Biostatistics, ACTREC, Tata Memorial Centre, for help with the statistical analysis.

\section{References}

1. Orringer D, Lau D, Khatri S, Zamora-Berridi GJ, Zhang K, Wu C, et al. Extent of resection in patients with glioblastoma: limiting factors, perception of resectability, and effect on survival. J Neurosurg 2012;117:851-859.

2. Stummer W, Pichlmeier U, Meinel T, Wiestler OD, Zanella F, Reulen
$\mathrm{HJ}$, et al. Fluorescence-guided surgery with 5-aminolevulinic acid for resection of malignant glioma: a randomised controlled multicentre phase III trial. Lancet Oncol 2006;7:392-401.

3. Acerbi F, Cavallo C, Broggi M, Cordella R, Anghileri E, Eoli M, et al. Fluorescein-guided surgery for malignant gliomas: a review. Neurosurg Rev 2014;37:547-557.

4. Moiyadi AV, Shetty PM, Mahajan A, Udare A, Sridhar E. Usefulness of three-dimensional navigable intraoperative ultrasound in resection of brain tumors with a special emphasis on malignant gliomas. Acta Neurochir (Wien) 2013;155:2217-2225.

5. Mair R, Heald J, Poeata I, Ivanov M. A practical grading system of ultrasonographic visibility for intracerebral lesions. Acta Neurochir (Wien) 2013;155:2293-2298

6. Sawaya R, Hammoud M, Schoppa D, Hess KR, Wu SZ, Shi WM, et al. Neurosurgical outcomes in a modern series of 400 craniotomies for treatment of parenchymal tumors. Neurosurgery 1998;42:10441055.

7. Moiyadi AV, Shetty P. Direct navigated 3D ultrasound for resection of brain tumors: a useful tool for intraoperative image guidance. Neurosurg Focus 2016;40:E5.

8. Belhawi SM, Hoefnagels FW, Baaijen JC, Aliaga ES, Reijneveld JC, Heimans JJ, et al. Early postoperative MRI overestimates residual tumour after resection of gliomas with no or minimal enhancement. Eur Radiol 2011;21:1526-1534.

9. Mohammadi AM, Sullivan TB, Barnett GH, Recinos V, Angelov L, Kamian K, et al. Use of high-field intraoperative magnetic resonance imaging to enhance the extent of resection of enhancing and nonenhancing gliomas. Neurosurgery 2014;74:339-348.

10. Unsgaard G, Selbekk T, Brostrup Muller T, Ommedal S, Torp SH, Myhr $\mathrm{G}$, et al. Ability of navigated 3D ultrasound to delineate gliomas and metastases: comparison of image interpretations with histopathology. Acta Neurochir (Wien) 2005;147:1259-1269.

11. Sanai N, Berger MS. Glioma extent of resection and its impact on patient outcome. Neurosurgery 2008:62:753-764.

12. Hervey-Jumper SL, Berger MS. Role of surgical resection in low- and high-grade gliomas. Curr Treat Options Neurol 2014;16:284.

13. Mert A, Kiesel B, Wohrer A, Martinez-Moreno M, Minchev G, Furtner J, et al. Introduction of a standardized multimodality image protocol for navigation-guided surgery of suspected low-grade gliomas. Neurosurg Focus 2015;38:E4.

14. Wilden JA, Voorhies J, Mosier KM, O'Neill DP, Cohen-Gadol AA. Strategies to maximize resection of complex, or high surgical risk, low-grade gliomas. Neurosurg Focus 2013;34:E5.

15. Incekara F, Olubiyi O, Ozdemir A, Lee T, Rigolo L, Golby A. The value of pre- and intraoperative adjuncts on the extent of resection of hemispheric low-grade gliomas: a retrospective analysis. J Neurol Surg A Cent Eur Neurosurg 2016;77:79-87.

16. Yordanova YN, Moritz-Gasser S, Duffau H. Awake surgery for WHO Grade II gliomas within "noneloquent" areas in the left dominant 
hemisphere: toward a "supratotal" resection. Clinical article. J Neurosurg 2011;115:232-239.

17. Solheim O, Selbekk T, Jakola AS, Unsgard G. Ultrasound-guided operations in unselected high-grade gliomas: overall results, impact of image quality and patient selection. Acta Neurochir (Wien) 2010;152:1873-1886.

18. Hammoud MA, Ligon BL, elSouki R, Shi WM, Schomer DF, Sawaya $R$. Use of intraoperative ultrasound for localizing tumors and determining the extent of resection: a comparative study with magnetic resonance imaging. J Neurosurg 1996;84:737-741.

19. Erdogan N, Tucer B, Mavili E, Menku A, Kurtsoy A. Ultrasound guidance in intracranial tumor resection: correlation with postoperative magnetic resonance findings. Acta Radiol 2005;46: 743-749.

20. Wang J, Duan YY, Liu X, Wang Y, Gao GD, Qin HZ, et al. Application of intraoperative ultrasonography for guiding microneurosurgical resection of small subcortical lesions. Korean J Radiol 2011;12:541546.

21. Petridis AK, Anokhin M, Vavruska J, Mahvash M, Scholz M. The value of intraoperative sonography in low grade glioma surgery. Clin Neurol Neurosurg 2015;131:64-68.

22. Coburger J, Scheuerle A, Thal DR, Engelke J, Hlavac M, Wirtz CR, et al. Linear array ultrasound in low-grade glioma surgery: histology- based assessment of accuracy in comparison to conventional intraoperative ultrasound and intraoperative MRI. Acta Neurochir (Wien) 2015;157:195-206.

23. Litofsky NS, Bauer AM, Kasper RS, Sullivan CM, Dabbous OH; Glioma Outcomes Project Investigators. Image-guided resection of high-grade glioma: patient selection factors and outcome. Neurosurg Focus 2006;20:E16.

24. Jakola AS, Berntsen EM, Christensen P, Gulati S, Unsgard G, Kvistad $K A$, et al. Surgically acquired deficits and diffusion weighted MRI changes after glioma resection: a matched case-control study with blinded neuroradiological assessment. PLoS One 2014;9:e101805.

25. Selbekk T, Jakola AS, Solheim O, Johansen TF, Lindseth F, Reinertsen I, et al. Ultrasound imaging in neurosurgery: approaches to minimize surgically induced image artefacts for improved resection control. Acta Neurochir (Wien) 2013;155:973-980.

26. Marko NF, Weil RJ, Schroeder JL, Lang FF, Suki D, Sawaya RE. Extent of resection of glioblastoma revisited: personalized survival modeling facilitates more accurate survival prediction and supports a maximum-safe-resection approach to surgery. J Clin Oncol 2014;32:774-782.

27. Southwell DG, Birk HS, Han SJ, Li J, Sall JW, Berger MS. Resection of gliomas deemed inoperable by neurosurgeons based on preoperative imaging studies. J Neurosurg 2018;129:567-575. 